Revue d'histoire de l'enfance « irrégulière »

Le Temps de l'histoire

$10 \mid 2008$

La prostitution des mineur(e)s au $X^{\mathrm{e}}$ siècle

\title{
Les yaouleds : entre marginalisation sociale et sédition politique
}

Retour sur une catégorie hybride de la casbah d'Alger dans les années 1930-1960

\section{OpenEdition}

\section{Journals}

Édition électronique

URL : http://journals.openedition.org/rhei/2917

DOI : 10.4000/rhei.2917

ISBN : 978-2-7535-1649-6

ISSN : $1777-540 \mathrm{X}$

Éditeur

Presses universitaires de Rennes

Édition imprimée

Date de publication : 1 octobre 2008

Pagination : 59-74

ISSN : 1287-2431

Référence électronique

Christelle Taraud, «Les yaouleds : entre marginalisation sociale et sédition politique », Revue d'histoire de l'enfance « irrégulière » [En ligne], 10 | 2008, mis en ligne le 26 septembre 2010, consulté le 03 décembre 2020. URL : http://journals.openedition.org/rhei/2917 ; DOI : https://doi.org/10.4000/rhei. 2917 


\section{Les yaouleds : entre marginalisation sociale et sédition politique}

\section{Retour sur une catégorie hybride de la casbah}

\section{d'Alger dans les années 1930-1960}

Enfants de la pauvreté, de la déstructuration des sociétés traditionnelles, de l'exode rural et de la naissance d'un sous-prolétariat dans les agglomérations urbaines coloniales, les yaouleds - de l'arabe ya (viens) et ouled (enfant) - sont aussi, dans une certaine mesure comme nous allons le voir, le produit de l'inter-communautarité sexuelle et de l'hybridation sociale. Entre le monde de la prostitution et la jeunesse explosive des anciennes casbahs déclassées et des nouvelles médinas indigènes à la périphérie desquelles vont bientôt fleurir les couronnes sans fin de bidonvilles -, il existe en effet un lien avéré et tangible : la figure ostentatoire du yaouled. Symbole colonial de la déchéance socioculturelle et de l'appauvrissement économique, le yaouled est le frère de la prostituée clandestine des bidonvilles et de la fille soumise " indigène " des quartiers réservés, mais c'est aussi son fils naturel et spirituel.(2)

Dans les années 1930, dans la casbah d'Alger, Lucienne Favre fait ainsi le portrait saisissant d'un de ces plus célèbres outlaws, Postillon, fils d'une prostituée kabyle et d'un convoyeur de bestiaux d'origine catalane - et ancien yaouled $-{ }^{(3)}$ qu'elle décrit en ces termes : «Son apparence était quelque peu théâtrale. D'immenses bottes, une culotte de cheval, un chandail de couleur vive, une casquette fortement rabattue sur le côté gauche pour cacher un œil crevé au cours d'une rixe, composaient son cos-
(1) Professeure dans les programmes parisiens de Columbia University et de New York University ; chercheuse à l'IRIS (laboratoire CNRS/EHESS) et à l'ULB (projet « Genre, normes et sexualité»).

(2) Cet article sur les yaouleds repose sur un long travail d'archives qui concerne plus largement la question de la prostitution et sur une analyse des sources secondaires (journaux, littérature, photographie et cinéma) qui éclairent, à mon sens, autant que les archives primaires - au demeurant bien silencieuses sur la question de la prostitution infantile féminine et mas-
Christelle

Taraud $^{(1)}$ culine dans l'Algérie coloniale - le sujet traité et analysé ici. Se référer cependant pour les sources primaires à Christelle Taraud, « De la mixité sexuelle : une rencontre coloniale ", La prostitution coloniale. Algérie, Tunisie, Maroc, I830-1962, Paris, Payot, 2003, p. 327-364 ;

et " Genre, classe et "race" en contexte colonial : une approche par la mixité sexuelle ", in Irène Théry, [dir], La dimension sexuée de la vie sociale, Paris, Éditions de l'EHESS, 2008.

(3) Le yaouled est autant - la précision est d'importance - un enfant errant (parfois orphelin) qu'un enfant des rues (ayant un ou des parents).

Christelle Taraud / p. 59 à 74 
(4) Lucienne Favre,

"Casbah. Un grand reportage sur la vie secrète des femmes d'Alger ", Voilà, 25 juin 1937. tume qu'il ne quittait ni de nuit ni de jour, attendant qu'il tombât naturellement en pourriture. "(4) Dans les villes coloniales en effet, le yaouled occupe toutes sortes de fonctions, notamment cireur de chaussure. Ce personnage, et son cri "Cirer M'siou, coup de brosse M'siou ", est un élément récurrent de la littérature et de la photographie coloniale. À titre d'exemple et pour bien montrer l'imprégnation de cette catégorie dans l'imaginaire collectif, ce poème anonyme intitulé "Le petit cireur» :

"Qu'il s'appelle Salah, Kacem ou Mohamed.

C'est le petit cireur sa boite en bandoulière.

Maigre sous des haillons ou sa blouse roulière.

Dont les siens ont quitté, jadis, l'ancestral bled.

Il est né citadin, à Bône ou Bab-el-Oued.

Mais la rue est pour lui partout hospitalière.

Qu'il s'appelle Salah, Kacem ou Mohamed.

C'est le petit cireur sa boite en bandoulière.

Il connaît sa ville, de son A jusqu'au $\mathrm{Z}$.

Il cire les souliers pour tâche journalière.

Mais en une pour tout de façon cavalière.

C'est Gavroche, Algérien, le petit yaouled.

Qu'il s'appelle Salah, Kacem ou Mohamed. "

"Gavroche méditerranéen, le yaouled est aussi commissionnaire (y compris dans les bordels), crieur de journaux, masseur de hammam, porteur de paniers, vendeur de cigarettes ou de chewing-gums, guide ou objet sexuel pour étrangers et locaux ; et peut devenir, une fois l'enfance passée, un proxénète d'autant plus aguerri aux lois du milieu qu'il a parfois grandi avec elles. C'est ce que laisse entendre un reportage réalisé, dans les années 1930, sur les "bas-fonds algériens ", publié dans le magasine illustré Voilà, édité par Gaston Gallimard. Dans la casbah, on appelle "magasins " les lieux où se prostituent les filles soumises dites "isolées " (elles sont cependant, comme les autres, "cartées » par la police des mœurs) qui peuvent, selon la réglementation, louer une pièce ou une chambre pour exercer leurs activités. Mme Ahmed qui, comme son nom ne l'indique pas, est d'origine espagnole et veuve d'un Arabe, est gérante de " magasins ». Autrement dit elle est chargée, chaque jour, de récolter les loyers auprès des filles. Elle a deux enfants métis 
dont un garçon qui semble avoir quelques dispositions particulières comme le raconte Lucienne Favre :

"Pour se concilier les bonnes grâces de la mère, les dames [les prostituées] leur font des cadeaux, leur sourient au passage. La petite est insignifiante, mais le fils promet d'être un dur. Parfois, le soir, au plein moment de la recette, il arrive en éclaireur précédant de peu madame sa mère et tapant du pied dans la porte des filles en hurlant des mots gras. Il a sept ans. Tout laisse prévoir que dans une dizaine d'années il pourra se substituer à sa génitrice. Les filles qui s'y connaissent en graine d'homme prévoient que ce petit donnera quelques soucis à leurs remplaçantes. " (5)

Remarquons que ce n'est pas la première description de ce genre particulier de maisons closes. En 1925, un article du Libertaire dénoncait déjà la condition des prostitués des "magasins":

"À treize ans, elles sont en "magasins”, dans un quartier nommé la Casbah, dans ces locaux infects où l'air et la lumière ne pénètrent jamais. Elles se livrent au premier venu pour la somme de un franc. Oui, vingt sous ! Certaines de ces femmes m’ont avoué avoir reçu jusqu’à trente clients dans la même journée. " (6)

Né dans la rue ou sur son seuil, dans un territoire "ensauvagé ", incontrôlable et violent, avec lequel il fait corps, le yaouled est enfin, comme nous le verrons, un émeutier en puissance qui réactualise à sa manière l'ancienne dissidence tribale, la sîba. Assoiffé de justice sociale, c'est aussi évidemment un client privilégié du nationalisme.

\section{Entre zinâ et fitnâ : "des enfants de la honte et du vice "}

Officiellement, comme le rappelle justement Emmanuelle Saada dans l'introduction de son livre Les enfants de la colonie. Les métis de l'Empire français entre sujétion et citoyenneté,(7) il n'y a pas de "question métisse " dans l'Algérie coloniale. La plus ancienne implantation française du second Empire colonial français, la plus proche de la " mère patrie ", aussi bien géographiquement que symboliquement, et la seule qui soit vraiment, à proprement parler, de peuplement - à l'exception notable, mais lointaine, de la Nouvelle-Calédonie -, aurait donc accouché, chose étrange et paradoxale, d'une société coloniale totalement étanche faisant fi des couples mixtes et, par extension, du produit
(5) Lucienne Favre,

"Casbah. Le grand reportage sur les bas-fonds d'Alger ", Voilà, 9 juillet 1937.

(6) Jane Maury, «La grande pitié des petites mauresques ou le bagne d'enfants de la Casbah ", Le Libertaire, 13 février 1925. Sur cette question, voir aussi Christelle Taraud, La prostitution coloniale..., op. cit.

(7) Emmanuelle Saada, Les enfants de la colonie. Les métis de l'Empire français entre sujétion et citoyenneté, Paris, La Découverte, 2007, 334 p. 
(8) Ainsi d'Émile Zola qui fait de Thérèse Raquin, l'héroïne de son livre éponyme publié en 1867 , la fille d'une Algérienne et d'un capitaine français nommé Degans.

(9) Émile Masqueray,

Souvenirs et visions d'Afrique, Paris, La Boîte à documents, 1989, p. 81-82. de ces unions (licites ou illicites) : les enfants métis, question secrète et occultée s'il en fut. Les archives sont en effet quasi muettes - a contrario de ce qui se passe dans le reste de l'Empire et tout particulièrement en Indochine où les documents sont légions - sur l'épineuse question des enfants métis nés dans l'Algérie coloniale. Au regard cependant des très nombreuses relations sexuelles ou amoureuses inter-communautaires, surtout visibles d'ailleurs dans les sources secondaires et notamment dans la littérature autobiographique ou de fiction, ${ }^{(8)}$ et de l'importance du peuplement européen - 700.000 dans l'ensemble du pays au début des années 1920 -, on s'explique mal cette absence récurrente de la figure du métis dans la société coloniale algérienne. On peut penser que des deux côtés de la frontière coloniale, on s'est accordé pour rendre invisible cette catégorie d'individus, probablement beaucoup plus nombreux qu'on ne le dit ou qu'on ne le croit sans pouvoir établir cependant avec précision les moindres statistiques. Cette invisibilité est d'autant plus facilement applicable au Maghreb qu'a contrario de ce qui se passe dans d'autres parties de l'Empire (et notamment en Indochine, en Nouvelle-Calédonie et en Afrique noire), le métissage, surtout quand il s'opère entre méditerranéens, peut très facilement passer inaperçu. Les souvenirs d'Émile Masqueray en témoignent. Voilà ainsi ce qu'il dit au fils de Morris, officier de la conquête de l'Algérie, et comment ce dernier lui répond :

"- Oui, dis-je, je pense à lui en vous regardant ; mais il y a chez vous quelque chose d'inexplicable.

- Quoi donc?

- La couleur ou l'expression de vos yeux.

- Vous ne saviez donc pas que ma mère est arabe ? me répond-il. " ${ }^{(9)}$ Du côté de l'administration coloniale, ce silence tient, d'abord et avant tout, à la peur d'un métissage qui aurait pu s'exercer au détriment de la " race " blanche et au bénéfice de l'islam. Dans les années 1830-1870, quelques unions - certes isolées mais ô combien symboliques, notamment quand elles concernaient des officiers ou des sous-officiers de l'armée d'Afrique - s'étaient soldées, en vertu du principe coranique interdisant aux femmes " indigènes " d'épouser des non-musulmans, par des conversions à l'islam et/ou par des mariages devant le cadi (le juge coutumier), qui avaient donné lieu ensuite à des reconnaissances, en bonne et due forme, d'enfants métis. Annie Rey 
Goldziguer signale ainsi les cas de De Neveu et Berbrugger bientôt suivis, nous dit-elle, par nombre d'officiers des bureaux arabes et notamment Morris, Martineau-Deschenez et Dargent. ${ }^{(10)}$ Après l'écroulement de l'Empire en 1870 et, avec lui, de l'idée chère à Napoléon III d'un Royaume arabe sous tutelle française, la vision des relations entre autochtones et colons change. Il ne s'agit plus de tolérer ni « fusion des communautés ", préconisée notamment par Pellissier de Reynaud, ${ }^{(1)}$ ni " mélange des races ", souhaité, malgré sa crainte d'une "dégénérescence de la race blanche ", par le comte Arthur de Gobineau dans son Essai sur l'inégalité des races humaines (I853-1855). ${ }^{(12)}$ Ces idées, discutées jusqu'alors au plus haut niveau de l'État métropolitain et colonial, n’ont plus droit de cité. Il s'agit désormais d'imposer une ségrégation raciale et communautaire qui vise à limiter au maximum la «contamination » et la "décivilisation » des Européens au contact des "indigènes ». Pensé, dès lors, dans le cadre d'une " compétition " raciale et confessionnelle ${ }^{(13)}$ encore complexifiée par la référence récurrente, chez les colonisés, à la " dégradation " et à la " corruption " des femmes "indigènes " au contact des nasrani (des chrétiens) -, le mariage mixte et licite était condamné à n'être, au cœur du dâr al-islam (mais aussi en métropole) et de la ségrégation coloniale, qu'un phénomène marginal fortement stigmatisé.

Ainsi, dans les années 1930, le docteur Georges Heuyer, fondateur de la pédopsychiatrie et pionnier du champ de "l'enfance inadaptée », souligne que « le nombre de métis issus de mariages d'Arabes et de Françaises est important " et qu'il convient de déterminer "s'il y a intérêt ou inconvénient à favoriser la venue d'indigènes coloniaux en France et leur mariage avec des Françaises ».
(10) Annie Rey Goldziguer, Le Royaume Arabe, Alger, Société nationale d'édition et de diffusion, 1977 , p. 74 .

(11) Eugène Pellissier de Reynaud préconise, pour favoriser le phénomène de l'endosmose, la généralisation des mariages mixtes afin de permettre une « fusion des communautés » qui verrait l'émergence d'un nouveau peuple, Annales algériennes, Paris, J. Dumaine, 1854, 464 p.

\section{(12) Arthur de}

Gobineau encourage en effet le " mélange des races " dans le but d'améliorer les « races inférieures ». Selon lui, l'attirance des Blancs pour les femmes "indigènes " s'expliquerait par leur " désir » de participer à cette " amélioration".
(13) Des voix s'élèvent, au début de la conquête, contre cette opposition tranchée islam/chrétienté. Ainsi le capitaine Richard - un officier des bureaux arabes polytechnicien et fouriériste qui s'exclame : « Nous avons des citoyens français qui sont juifs, protestants, catholiques ; pourquoi n'ajouterions-nous pas à cette liste des musulmans?" 
(14) Georges Heuyer et

\begin{abstract}
Françoise Lautmann,
«Troubles du caractère

et inadaptation sociale

chez les enfants métis ",

Archives de médecine

des enfants, tome 40 ,

$\mathrm{n}^{\circ} 9$, septembre 1937 ,

p. 553-564.
\end{abstract}

(15) Guy Pervillé, Les étudiants algériens de

l'université française, I8801962, populisme et nationalisme chez les étudiants et les intellectuels musulmans algériens de formation française, Paris, Éditions CNRS, 1984, p. 91.

(16) Ainsi dans la Régence d'Alger, les Kulughli (métis nés d'un père turc et d'une mère " indigène ») avaient-ils toute leur place, y compris dans le jeu politique... mais bien sûr, ces hommes étaient musulmans.
(17) Cité dans Yvonne Knibiehler, Geneviève Émmery et Françoise Leguay, Des Français au Maroc : la présence et la mémoire, Paris, Denoël, 1992, p. 80-81.

(18) En 1830, après la victoire des Français, l'acte de reddition du Dey d'Alger stipule que «l'exercice de la religion restera libre ; la liberté de toutes les classes d'habitants, leur religion, leurs propriétés, leur commerce, leur industrie, ne recevront aucune atteinte. Les femmes seront respectées ». Cité par Daniel Rivet, Le Maghreb à l'épreuve de la colonisation, Paris, Hachette, 2002, p. 112.
Derrière tout cela se trouve la question centrale de "l'adaptation sociale des métis " en métropole. ${ }^{(14)}$ À noter aussi que les mariages mixtes femmes blanches/« indigènes " ne sont pas mieux acceptés de l'autre côté de la frontière coloniale. Dans les années 1930 toujours, en Tunisie, les "indigènes " qui prennent femme chez l'occupant n'ont ainsi plus le droit d'être enterrés au cimetière musulman. Et, en 1934, l'Association des étudiants musulmans nord-africains condamne les mariages mixtes que ses adhérents pourraient contracter en France. ${ }^{(15)}$

Le rejet du mariage mixte, comme forme classique, dans la région, ${ }^{(16)}$ de régulation de la sexualité intercommunautaire et de l'enracinement, dans la société, d'un pouvoir acquis par la force ${ }^{(17)}$ - comme l'explique d'ailleurs un Marocain à un contrôleur civil : « Les Français pour nous, c'étaient des envahisseurs, plus forts que nous, bien armés, calculant tout, réfléchissant à tout. On a cru qu'ils allaient dominer le pays et se donner comme les nouveaux chefs. Ils allaient épouser les filles des meilleures familles de la contrée. S'ils avaient vraiment fait cela, ils auraient dominé les tribus " - a alors logiquement débouché sur le développement d'une marginalité sexuelle (notamment prostitutionnelle). Marginalité qui, tout en traduisant évidemment le droit du conquérant, était aussi le produit d'un statu quo (une majorité de femmes préservée contre une minorité sacrifiée) ${ }^{(18)}$ globalement accepté, dès la fin du XIXème siècle, par l'ensemble des hommes, "indigènes " et Européens. En somme, bien que les nasrani (les chrétiens) aient renversé un statut séculaire - celui de dhimmi (en terre d'islam, les chrétiens et les juifs sont des "protégés ") - et qu'ils aient réduit l'un des tabous les plus prégnants de la sexualité précoloniale, 
celui de la ségrégation confessionnelle du sexe, ${ }^{(19)}$ le " partage » des femmes, pourtant nécessaire puisque le sex ratio s'exerçait toujours à leur désavantage, restait problématique. La majorité des musulmanes étant intouchables, l'administration coloniale utilise alors une population féminine de «l'entre-deux " qu'elle constitue, en raison de sa nature même, en minorité sexuelle dominée. L'imposition du stigmate prostitutionnel - l'égout séminal conceptualisé au XIXème siècle, en métropole, par le docteur réglementariste Alexandre Parent-Duchâtelet - a, en effet, consubstantiellement agrégé, en contradiction avec le fantasme récurrent des colonies comme "édens sexuels ", la sexualité vénale algérienne au monde de la marginalité sexuelle. Ce faisant, l'administration coloniale réglait en sus l'épineux problème de la filiation et de la citoyenneté - c'est-à-dire des enfants métis qui auraient pu naître de ces unions illégitimes. Enfants de prostituées « indigènes » avant tout, ces derniers sont renvoyés, du fait du double statut discriminant de leurs mères, à la « honte " et au " vice " de leur naissance.

Dans ce contexte, on ne s'étonne nullement que la " question métisse » ne soit plus abordée, du côté français - quand elle l'est, ce qui est bien rare comme nous l'avons déjà souligné - qu'en faisant des métis, enfants bâtards et hybrides des "classes laborieuses, dangereuses et vicieuses ", des " hors castes » et des "parias " (triplement étrangers à eux-mêmes au regard de la " race ", de la religion et de la société dans laquelle ils ont vu le jour) dont la frustration et le ressentiment sont d'autant plus inquiétants qu'ils sont perçus comme une menace latente contre l'ordre colonial. Ce sont en effet essentiellement des enfants métis non reconnus par leur père français (et donc assimilés automatiquement au statut indigène de leur mère) qui sont objets de débats. Ceux-là même que l'on retrouve ensuite dans la catégorie englobante de yaouleds, même si tous les enfants des rues ne sont pas, évidemment et loin s'en faut, des enfants illégitimes d'unions inter-communautaires illicites. Du point de vue des colonisés, cette partie spécifique des yaouleds (enfants naturels et hybrides) n'est cependant - à l'image de leurs mères "partagées " et donc "dégradées " - guère moins problématique que pour les Européens. Entre zinâ (sédition sociale et morale) et fîtnâ (sédition politique), ils imposent l'image troublée d'une société " indigène " fracturée et déliquescente. A fortiori quand ces yaouleds (métis ou non) remettent à leur
(19) À la veille de

l'intervention militaire française à Alger, les femmes libres de confession musulmane, surprises avec des chrétiens ou des juifs, étaient enfermées dans des sacs et jetées à la mer. C'est dire la force du tabou religieux (harâm) brisé par la présence coloniale. 
(20) Cette partie de

l'article est tirée du seul sous-chapitre non publié de ma thèse de doctorat intitulé «Aux marges de la prostitution clandestine : maricones et gigolos ", Prostitution et colonisation. Algérie,

Tunisie, Maroc, I830-1962, thèse d'histoire, Paris I, 2002, p. 198-202.

(21) Sur cette question, voir Christelle Taraud, «Une marginalité révélée à la ville ", La prostitution coloniale..., op. cit p. $198-207$. tour en cause l'ordre colonial et local par une sexualité problématique (parce qu'exercée avec des hommes de " race » et de confession différentes).

\section{Les yaouleds et le commerce du sexe ${ }^{(20)}$}

Le 13 février 1925, Jane Maury écrit ainsi dans Le Libertaire un article intitulé « La grande pitié des petites mauresques ou le bagne d'enfants de la Casbah » où elle souligne, scandalisée :

«De retour d'Alger, outré de ce que j'ai vu, je tiens à vous le faire connaître [...] Je veux vous dire la vie des malheureuses mauresques qui font le commerce de leur corps. Elles sont, hélas, en très grand nombre. Que sontelles? Des filles perdues par le vice et la débauche ? Non ! Perdues par la misère et la souffrance. La plupart orphelines, les autres abandonnées par leurs parents qui ne peuvent les nourrir. L'Assistance publique qui fonctionne très mal pour les Européens, ne fonctionne pas du tout pour les indigènes. Un père, une mère meurt, ils laissent des enfants qui sont abandonnés. Pour les garçons, passe encore, ils cirent les chaussures, ouvrent les portières, souvent ont des coups en guise de paiement, mais arrivent tant bien que mal à payer leur croûte et, le soir venu, pour vingt sous, vont coucher au bain maure. Mais les filles, que deviennent-elles sur le pavé ?"

Les filles deviennent souvent prostituées après avoir tenté, au préalable, de vivre du produit de leur travail en se plaçant notamment comme domestiques dans les familles riches de la médina ou de la ville européenne. ${ }^{(21)}$ Mais J. Maury évacue cependant un peu rapidement la question des relations sexuelles monnayées que ces jeunes garçons pourraient avoir dans le but d'assurer leur subsistance en sous-entendant que eux, a contrario des filles, peuvent plus facilement s'insérer dans l'économie parallèle des «petits métiers ".

Il semble assez logique cependant, au regard du mode de vie des yaouleds, de s'interroger sur leur rapport avec le commerce du sexe. Beaucoup de ces jeunes garçons traînent, travaillent, vivent et dorment en effet dans les rues de la casbah et de la ville européenne, de jour comme de nuit, et peuvent facilement y rencontrer des clients (de passage ou habitués) locaux ou Européens avec lesquels ils auront des relations sexuelles monnayées. Ces relations sont d'autant plus facilement concevables et réalisables que les yaouleds bénéficient, une fois n'est pas coutume, de leur statut d'enfant ou de jeune garçon - statut 
qui les préserve, dans les sociétés locales, du déshonneur viril où les conduirait le fait « de coucher avec des hommes " une fois devenus adultes. Enfants ou adolescents « errants " soumis à la sexualité dominatrice - en général, ils sont perçus comme « passifs » - et parfois violente ${ }^{(22)}$ des adultes qui les rétribuent pour leurs faveurs, les yaouleds sont donc souvent, au même titre que les filles, des prostitués en puissance. Dans un autre contexte qui concerne aussi de jeunes enfants pouvant venir de la rue, celui des corporations artisanales, le maitre (tafar: actif) entretient parfois des relations " homosexuelles " (23) institutionnalisées ${ }^{(24)}$ avec son disciple (miboun : passif) garçon qui lui est confié par la famille ou orphelin qu'il prend en charge de lui-même.

Lucienne Favre en témoigne encore simplement en 1937 en brossant le tableau suivant d'Aïssa, boutiquier mozabite de la rue Randon dans la casbah d'Alger : «En attendant, loin de sa femme rentrée au M'Zab, comme le veut la coutume, il mène en compagnie d'un jeune disciple ${ }^{(25)}$ au regard de velours, aux jambes torves, à la voix mélodieuse qui le sert à toute heure du jour, à toute heure de nuit, une vie épicurienne. " (26) Apparemment Aïssa possède une vie familiale "normale " parfaitement compatible avec sa situation et ne se cache pas d'entretenir des relations "particulières " avec son disciple. ${ }^{(27)}$ Cela se sait dans la casbah et ne donne pas lieu à réprobation ou à sanction sauf si l'une des deux parties (généralement le disciple) s'avise de donner des signes trop évidents de "féminité " et de s'installer durablement dans une situation de passivité sexuelle qui irait au-delà de sa condition. D'ailleurs, l'état transitoire de la situation (on ne reste pas disciple, comme on n'est pas yaouled, toute sa vie) et le caractère non exclusif de la relation (le maitre est souvent marié et parfois polygame) sont des
(22) On ne sait rien,

par exemple, des violences sexuelles et des viols que ces enfants peuvent subir dans la rue. Les archives sont, à ma connaissance, muettes sur cette question et les témoignages littéraires bien peu parlants.

(23) Je mets ici le terme homosexuel entre guillemets à dessein pour signifier des relations sexuelles entre hommes et non une identité homosexuelle perçue et pensée en tant que telle-

c'est-à-dire sur un mode qui renverrait à la prétendue " modernité sexuelle " du monde occidental d'aujourd'hui constituant, outre un anachronisme, une forme d'européocentrisme extrêmement préjudiciable à

l'analyse des relations (y compris prostitutionnelles) entre hommes.
(24) Cette assertion est aussi valable pour le Seigneur (Sidnâ) et pour le Roi (Mâlik) qui ont des « mignons qu'on voit et qu'on chante, qu'on affiche et dont on tire vanité ", Émile Mauchamp, La sorcellerie au Maroc, Paris, Dorbon Ainé, (s.d.), p. 167.

(25) En arabe, on dit aussi ghoulam, c'est-à-dire en même temps jeune serviteur et giton.

(26) Lucienne Favre, op. cit., p. 16.

(27) Il ne serait pas étonnant qu'il fréquente, de surcroît, quelques filles de joie. 
(28) Georges Lapassade,

"Homosexualité ethnique ou amour vrai ?", in Zakya Daoud, [dir.],

« Les maladies francoarabes de $\mathrm{A}$ jusqu'à $\mathrm{Z}$ ", Panoramiques, $\mathrm{n}^{\circ} 3$, premier trimestre 1992, p. 95.

(29) Christelle Taraud, La prostitution coloniale..., op. cit.

(30) André Gide, L'Immoraliste, Paris, Flammarion, 1902, p. 13. gages d'absence de « déviation sexuelle ». En revanche, si la pratique " homosexuelle " perdure postérieurement à ce cadre singulier, le sujet apparait, dans la société locale, soit porteur d'une " maladie " (assimilable à une possession par un djoun, par un génie), soit vecteur d'une " perversion sociale " (zîna) inacceptable. Dans les deux cas, il renonce, ce qui est socialement incompréhensible, à sa virilité (puisqu'il s'installe dans un rôle de "femme ») et aux privilèges conséquents qui l'accompagnent et assurent sa domination « naturelle ». C'est ce que Georges Lapassade appelle, à mon sens un peu abruptement, la transition entre "l'homosexualité ethnique » et "l'homosexualité psychologique ». (28) Proche du "vrai amour », "l'homosexualité psychologique " se distinguerait alors de "l'homosexualité ethnique " par son caractère " civilisé ", créant ainsi une évidente hiérarchie entre la " bonne " et la « mauvaise » manifestation sociale de l'homosexualité. Quoi qu'il en soit cependant ce débat étant loin d'être tranché y compris par la sociologie et l'anthropologie contemporaines -, dans une société sexuellement ségréguée, "l'homosexualité ethnique " (au même titre que la prostitution féminine ${ }^{(29)}$ ) propose donc un mode de régulation de la sexualité reposant sur une disponibilité contrôlée et transitoire (liée à une classe d'âge ou à un statut social transitoire ou marginal).

La sexualité monnayée des yaouleds s'inscrit, du moins quand elle se passe entre hommes de même origine et de même confession, dans le même phénomène et est acceptée tant qu'elle ne franchit pas les règles du harâm (ce qui est interdit) et de la hachma (de la honte et de la pudeur). Là se construit une partie du malentendu avec les Occidentaux qui croient arriver dans " un no man's land primitif ", un " éden homosexuel » sans règles, ni tabous. Une vision exotique et romanesque, largement portée par des auteurs comme Jean Genet, William Burroughs ou André Gide qui fait la description suivante du petit biskri Bachir, personnage fantasmatique de son roman L'Immoraliste : " La gandourah, un peu tombée, découvre sa mignonne épaule. J'ai besoin de le toucher. Je me penche. Il se retourne et me sourit. " ${ }^{(30)}$ À cette vision se surexpose l'illusion d'une relation réciproque et égalitaire, d'un "véritable amour ", qui pourrait se construire au-delà de la domination coloniale, du commerce du sexe et de l'industrie du tourisme. C'est pourtant bien la convergence de ces deux facteurs consubstantiellement imbriqués (« dispo- 
nibilité sexuelle » des « indigènes » créée par la paupérisation coloniale et possibilité de relations sexuelles " monnayées " ouvertes par l'instauration d'une industrie du sexe) qui permet le développement d'un nouveau marché concurrentiel, alors encore en formation. Il s'agit du tourisme prostitutionnel homosexuel, ${ }^{(31)}$ dans lequel la figure du yaouled prend évidemment toute sa place. Commerce sexuel vécu, en Algérie, comme un élément supplémentaire de corruption de l'Occident, comme une "impropreté morale " totalement synonymique du système colonial qui pourrait se résumer à la formule lapidaire suivante : "Ils viennent ici pour user sexuellement de nos enfants. »

Marino Zermac, qui se trouve à Alger après la seconde guerre mondiale, souligne sans détours cet état de fait dans ses mémoires :

"Alger, sa vie grouillante, son mélange de races, d'idiomes, de costumes, est pour moi un régal. J'aime aussi ses odeurs d'épices, ses chants, ses mélopées, le linge multicolore séchant aux fenêtres, le bruissement du vent dans les palmiers. Ici, le spectacle est dans la rue... Pierre est ravi. Les petits mendiants nous offrent leurs services pour quelques sous et, comme nous restons de marbre devant leurs suggestions, ils nous proposent leurs petites sœurs... pour encore moins cher [...]. Dans ces années d'après-guerre, Alger était la ville du sexe comme le sont devenus Bangkok, Manille, Cuba ou Rio de Janeiro. Les enfants y étaient beaux, pas farouches et se prostituaient pour très peu d'argent. Le voyage était encore réservé aux personnes fortunées, on ne parlait pas encore de tourisme sexuel. Mais tout ce que la vieille Europe comptait de pédophiles accourait en Algérie pour donner libre cours à ses vices. Les grands artistes homosexuels d'André Gide à Henry de Montherlant passaient chaque année quelques semaines en Algérie sous prétexte de "voyages d'études". D’ailleurs, les mœurs des autochtones s'y prêtaient. Si la famille musulmane était généralement exemplaire, élevait bien ses enfants, les maintenait sur le bon chemin, la misère venait trop souvent perturber ce bel ordre ancestral. Et puis beaucoup d'hommes étaient morts à la guerre laissant leur famille dans le dénuement. Le yaouled représentait pour les pédés ce que la petite bretonne était pour l'amateur de chair fraîche parisien. " (32)

Vivant en partie de la prostitution (comme prostitués, commissionnaires dans les bordels ou futurs proxénètes), les yaouleds sont donc intimement liés
(31) Plus tard, dans les années 1980 , on ira même jusqu’à parler des " charters de folles " à destination des pays dits " exotiques ", Georges Lapassade, op. cit.

(32) Marino Zermac, Une vie sans importance. Voir le site :

www.apophtegme.com/ ZERMAC/zermac06.htm 
(33) Carlier (Omar)

Entre nation et jihad. Histoire sociale des radicalismes algériens, Paris, Presses de Sciences-Po, $1995,443 \mathrm{p}$ au commerce du sexe. Ils en connaissent les agents, les rouages et les territoires - le caractère violent aussi. Enfants de la misère économique (ils sont liés à la petite criminalité et survivent fréquemment grâce aux larcins qu'ils commettent) et de la fracture identitaire, ils sont aussi souvent au moment de la guerre de libération, comme nous allons le voir maintenant au travers de La bataille d'Alger, des acteurs du nationalisme algérien : agents de liaison, agitateurs des masses, porte-paroles de la politique du FLN et parfois aussi " assassins " à la solde du parti. À noter, en préambule, que j’utilise ici le film La bataille d'Alger comme révélateur d'une situation sociale et politique (le nationalisme algérien et ses liens avec le monde de la rue et de la déviance sexuelle) à laquelle la jeunesse algérienne est confrontée et à laquelle elle participe. Bien que film, La bataille d'Alger exemplifie, à mon sens parfaitement, ce que de très nombreux livres sur la guerre d'Algérie confirment par ailleurs et notamment celui d'Omar Carlier ${ }^{(33)}$ qui, en ce domaine, est incontournable, les liens entre la jeunesse explosive des villes et le nationalisme (visible dans la figure paradigmatique d'Ali la Pointe). Le fait que La bataille d'Alger soit de plus clairement un film de propagande (Yacef Saadi étant à l'origine d'un scénario reposant en grande partie sur ses mémoires) accentue encore l'idée que le discours qui y est véhiculé procède, au même titre qu'un tract ou qu'un communiqué du FLN, de l'énoncé du projet politique et de la place que chacun doit y trouver. C'est pourquoi il m'a semblé particulièrement utile et intéressant - au même titre que ce que j'ai fait avec Pépé le Moko et La Bandera dans La prostitution coloniale - d'utiliser La bataille d'Alger comme "source primaire " éclairant la position du FLN sur les questions traitées dans cet article et tout particulièrement sur les liens entre déviance, sexualité illicite et nationalisme.

\section{La bataille d'Alger : des yaouleds en action}

La bataille d'Alger commence officiellement le 7 janvier 1957 quand les soldats français commandés par le général Massu investissent massivement la casbah. Elle se termine le 8 octobre 1957 avec la mort d'Ali la Pointe, dernier chef libre de la fédération du FLN à Alger. Ali la Pointe est né le 14 mai 1930 et il connaît très tôt la misère. À 13 ans, il se retrouve de surcroît en prison. Libéré, il se rend à Alger où il s'inscrit dans un club de boxe de Bab- 
el-Oued, tout en suivant une formation en maçonnerie. Deux incidents vont le rendre encore plus " rebelle » au pouvoir colonial et attiser sa soif de vengeance : une gifle assenée par un policier français et une correction qu'il a administrée à un ressortissant européen, lui valent, à l'âge de 22 ans, une condamnation aux travaux forcés et une incarcération à la prison de Damiette, dans la wilaya de Médéa. Il s'en évade le 2 avril 1955 et se rend à Blida puis à Alger où il rentre dans la clandestinité et combat aux côtés de Yacef Saâdi, le chef de la fédération du FLN à Alger. Dans La bataille d'Algerfilm interdit en France à sa sortie en 1966 -, cet ancien yaouled devient le héros de l'un des épisodes les plus importants et symboliques de la guerre d'Algérie et, par extension, de la mythologie nationale algérienne. Guérillero urbain (fidaì) et figure emblématique de la bataille d'Alger, le chahid (martyr) meurt le 8 octobre 1957 dans l'explosion de sa cache avec deux de ses compagnons de lutte, un enfant de douze ans, Omar bou Hamadi, et une femme combattante, Hassiba ben Bouali.

Au travers de son destin hyperbolique, la figure d'Ali la Pointe traduit un changement fondamental et profond dans le rôle que les yaouleds vont prendre dans la vie politique algérienne et tout particulièrement dans la révolution nationale. ${ }^{34)}$ Dans l'ensemble du film en effet, ces derniers vont œuvrer sans relâche pour faire triompher les idéaux du Front de libération nationale. Se fondant dans la masse parce que figures ordinaires de la médina et des quartiers métissés de la ville européenne (à l'image de Bal-el-Oued où ils sont chez eux), les yaouleds se glissent dans les interstices de la ville d'autant plus facilement que leur statut d'enfant semble les protéger globalement de la vindicte des Européens en guerre. Au journal de $20 \mathrm{~h} 00$ du 28 janvier 1960, diffusé par l'ORTF alors qu'Alger se trouve au cinquième jour de la semaine des barricades organisée par les partisans de l'Algérie française, des images saisissantes nous montrent des soldats français qui, pendant un moment de détente, lisent le journal debout contre un tank et se font cirer les chaussures par deux ouled-plaça (enfants errants) de la place du gouvernement à Alger. ${ }^{(35)}$ Dans le film de Gillo Pontercorvo, on se trouve bien loin de cette image sereine et rassurante des petits cireurs de chaussures encore " au service " des agents du pouvoir colonial. Les enfants des rues de la casbah se retrouvent, tout au contraire, aux avant-postes de la lutte contre l'ordre colonial qu'il s'agisse
(34) Voir Christelle

Taraud, « De la mixité... ", op. cit, p. 327364 ; et Omar Carlier, "Classe d'âge et citadinité ", " Les lieux du politique » et «Violence coloniale et mystique insurrectionelle ", Entre nation et jihad, op. cit., p. 32-77, p. 164205, p. 271-311.

(35) Archives de l'INA. 
(36) Pour une comparaison avec le Maroc, voir l'article de Bruno de Rotalier, "Les yaouleds (enfants des rues) de Casablanca et leur participation aux émeutes de décembre 1951 ", le Temps de l'histoire. Revue d'histoire de l'enfance "irrégulière", n 4, 2004.

(37) C'est aussi Omar qui est placé au cour de l'action du film puisque c'est lui qui est chargé de remettre le premier message du FLN à Ali la Pointe - message qui lui indique ce qu'il doit faire (en l'occurrence tuer un policier français) pour rejoindre le FLN.

(38) À l'image de ses tatouages. Sa fiche d'identification policière signale ainsi : Tatouage «Zoubida - Choda Felah " sur main gauche, « Marche ou crève » sur téton gauche et " Tais toi » dessus pied droit. de délivrer des messages à des agents du FLN, de leur livrer des armes ou bien de se mettre au service du discours nationaliste. ${ }^{(36)}$ À cet égard, le rôle joué dans le film par le petit Omar est très symptomatique de cette véritable « mue du yaouled " au contact d'un idéal patriotique visant à construire, collectivement, l'umma wataniyya (la communauté nationale). Constamment présent dans les moments-clés de La bataille d'Alger, ${ }^{(37)}$ c'est lui qui incarne tout à la fois le renouveau (comme représentant d'une nouvelle génération, massive en nombre, se levant contre le colonialisme) de l'orgueil national et la perpétuation de la lutte (alors même que les adultes, hommes et femmes confondus, semblent assommés par la violence de l'opération de "pacification " réalisée par les parachutistes dans la casbah). Prenant le micro laissé vaquant par un gendarme français chargé de la propagande anti-FLN au moment du retour des premiers hommes arrêtés et relâchés car n'appartenant pas à la fédération du FLN d'Alger, Omar harangue ainsi les Algériens et les Algériennes présents : "Frères, frères, écoutez bien. Le FLN vous demande de ne pas avoir peur. La grève a été un succès. L’organisation est avec vous. "Ce à quoi les hommes et les femmes présents répondent par un "Vive l'Algérie ", scandé à de multiples reprises sur fond de youyous...

Entre le petit Omar et Ali la Pointe il y a donc un lien évident : celui de la pauvreté et de la révolte contre l'humiliation et l'injustice - lien qui les a tous les deux conduits au nationalisme. Jusqu'à son engagement au sein du FLN, Ali la Pointe - qui est, comme de nombreux Algériens de l'époque, complètement analphabète - était en effet un simple petit malfrat multirécidiviste lié au milieu du jeu et de la prostitution. ${ }^{(38)}$ À travers lui cependant, ce que montre bien le film, ce n'est pas seulement la reprise en main politique de la casbah mais aussi sa moralisation. (39) Avant le déclenchement de la bataille d'Alger, un communiqué du FLN d'avril 1956 avait déjà clairement mis en avant le lien entre politisation et moralisation :

"Peuple algérien, l'administration coloniale n'est pas seulement responsable de la misère de notre peuple et de son esclavage mais aussi de l'abrutissement, de la corruption et des vices dégradants de beaucoup de nos frères et sœurs qui ont oublié leur propre dignité. Le Front de libération nationale engage une action pour extirper tous ces fléaux et appelle toute la population à l'aider par son concours car c'est une condition première 
pour obtenir l'indépendance. À partir de ce jour, les autorités clandestines du FLN assument la responsabilité de la santé physique et morale du peuple algérien et décide en conséquence : sont interdits la consommation et la vente de tous types de drogues et de boissons alcoolisées, sont interdits la prostitution et le proxénétisme. Les contrevenants seront punis, les récidivistes seront punis de la peine de mort. ".

La mise en pratique de cette politique est visible dans trois moments phares du film dont deux concernent directement Ali la Pointe : ce dernier frappe d'abord dans un café maure, tout en le menaçant violemment, un fumeur de kif ; puis se rend ensuite dans une des rues chaudes de la casbah (la rue Barberousse ou la rue Kattaroudjil) - en interrogeant au passage les prostituées de magasins et celles de maisons (qu'il semble très bien connaître) - pour assassiner un proxénète notoire, Hocene dont il était l'ami avant d'entrer au FLN. En le tuant d'une rafale de mitrailleuse, Ali interpelle les deux acolytes d'Hocene en ces termes : "Désormais les choses vont changer dans la casbah. Nous allons nettoyer le pays de cette saleté ! "Dans le même ordre d'idée, la scène de l'ivrogne de La bataille d'Alger est très éclairante. D'abord invectivé en arabe par une femme en hä̈ck, un homme extrêmement aviné est ensuite agressé par une foule d'enfants rameutés par Omar. Ce sont eux qui lui font dévaler l'un des escaliers de la casbah en le traînant par terre, en le rouant de coups et en l'insultant ( Alcoolique! Alcoolique!»). Emblématique du film, cette scène est aussi symbolique, à mon sens, de la nature de la nation algérienne en construction.

Cette "Algérie virile » qui s'exprime dans le cadre de la lutte pour l'indépendance - fabrique des héros et des martyrs par excellence à l'image de la mort d'Ali la Pointe et du petit $\mathrm{Omar}^{(40)}$ - marque donc une régénération de la population à partir d'une de ces catégories les plus problématiques, les yaouleds ; et impose ensuite, dans l'État post-colonial, un projet politique reposant sur une normalisation, une uniformisation et une moralisation de la société algérienne ${ }^{(41)}$ qui se fait, entre honneur viril et honneur national ${ }^{(42)}$ au détriment de la pluralité politique, sociale et confessionnelle de la nation. Dans cette grande entreprise de nationalisation des Algériens et des Algériennes, qui s'effectue sur la base d'une morale unique encadrée par un parti unique, ${ }^{(43)}$ tout ce qui faisait désordre dans la société coloniale doit être désor-
(39) Voir Christelle Taraud, « De la mixité... ", op. cit.; et Omar Carlier, op. cit.

(40) Ces derniers invités à se rendre par l'armée française préféreront mourir dans l'explosion de leur cache.

(41) Voir Christelle Taraud, "De la mixité... ", op. cit. ; et Omar Carlier, op. cit.

(42) Se posent dans ce cadre notamment les questions de la collaboration charnelle et de l'aliénation à l'Occident (donc à la colonisation). Sur ces problèmes, voir Christelle Taraud, La prostitution coloniale... op. cit., p. 327-363.

(43) À l'image de la scène de mariage de La bataille d'Alger qui se fait sous la haute conduite d'un cadre politique du FLN. Sur cette question des mariages au sein 
de l'organisation, voir les livres de : Djamila Amrane, Les femmes dans la guerre d'Algérie, Paris, Plon, 1991, 298 p. ;

Diane Sambron, Femmes musulmanes. Guerre d'Algérie (1954-I962), Paris, Autrement, 2007, 194 p.

(44) Au Maghreb, à

l'époque, la position de garçon de café (garsonat) est souvent suspectée de permettre la rencontre de clients.

Le garsonat fait donc aussi partie du milieu de la prostitution clandestine. mais éradiqué : prostituées inter-communautaires et yaouleds hybrides et en guenilles notamment. Comme symboles d'une société clochardisée et métissée, mais aussi comme représentants de l'humiliation collective de la nation et de l'être algériens (le traumatisme collectif que représentent des yaouleds cirant et lustrant les chaussures des "blancs ", acte perçu par excellence comme « servile ", est extrêmement vivace à l'époque), ces derniers sont donc condamnés à disparaître. En 1963, le président de la République algérienne, Ahmed ben Bella, décide d'ailleurs fort symboliquement de vider les rues de la casbah (et par extension du pays) de ses yaouleds en mettant en place un vaste programme éducatif en leur faveur - programme dont on ne connaîtra jamais vraiment le bilan au demeurant. Anecdote intéressante, on prétend qu'Ahmed ben Bella aurait décidé de la mise en place de ce programme après avoir écouté, en 1963, le grand chanteur de chaâbi Amraoui Missoum interpréter Des roses blanches pour ma mère - chanson dédiée à la dramatique histoire d'un jeune cireur de chaussures. Cette chanson aurait eu d'autant plus d'impact sur le président que Amraoui Missoum, né dans la casbah en 1921, était lui-même un enfant de la misère qui avait exercé tous les métiers de l'indigénat et avait notamment été cireur de chaussures et garçon de café... ${ }^{(44)}$

Ce faisant, il rejette dans l'ombre cette histoire complexe et ambiguë, liant dans un même mouvement mixité sexuelle et prostitution (féminine et masculine), jeunesse déclassée et nationalisme, assimilation et identité hybride, dont les yaouleds étaient les produits volontaires ou forcés et que Kateb Yacine avait su si bien rendre dans son livre Nedjma. Nedjma - la nation algérienne, la femme-patrie par excellence -, fille métisse d'une Française « légère » et de deux de ses amants algériens qui atteste pourtant de la présence symbiotique de l'autre en soi. Une présence trouble, parce que fortement sexualisée, et donc problématique qu'on rejette avec force à partir de 1962 et qui pourtant continue encore aujourd'hui à travailler le social et le politique. C'est de cette présence dont les yaouleds témoignent aussi malgré eux et c'est pourquoi il est plus confortable, ici et là-bas, de les reléguer dans l'oubli des mémoires et de l'histoire. 